\title{
Ectodermal Dysplasia Syndrome with Orthodontic Treatment and Oral Finds: A Case Report
}

\author{
Weihao $\mathrm{Li}^{1}$, Yanhua $\mathrm{Xu}^{2 *}$ and Song $\mathrm{Li}^{3}$ \\ ${ }^{1}$ MD., School of Stomatology, Kunming Medical University, Kunming, China. \\ ${ }^{2}$ Professor, Department of Orthodontics, School and Hospital of Stomatology, Kunming Medical University 650101, \\ Kunming, China. \\ ${ }^{3}$ Professor, School of Stomatology, Kunming Medical University, Kunming, China.
}

Received: September 7, 2017; Accepted: October 4, 2017; Published: October 31, 2017

*Corresponding author: Yanhua Xu, PhD, Professor, Department of Orthodontics, School and Hospital of Stomatology, Kunming Medical University, 650101, Kunming, China. Tel: +86 13354941072; E-mail address: xuyanhua18@163.com

\section{Abstract}

Ectodermal dysplasia, which affects ectodemal derivatives, currently is an X-linked recessive inheritary hypohydrotic ectodermal dysplasia. Patients with ectodermal dysplasia usually characterized by congenital teeth loss, abnormal teeth and inherited through female carriers. In this case report, we present a 5 years old boy of China ethnicity with mandibular anodontia, a rare dental dysplasia in ectodermal dysplasia syndrome. To compromise esthetics and function, a multidisciplinary approach was performed by oral physician, oral pathologist, orthodontist and prosthodontist.
\end{abstract}

Keywords: Ectodermal dysplasia; Multidisciplinary; Orthodontics;

\section{Introduction}

Ectodermal dysplasia, a hereditary disorder, manifests developmental dystrophies but normal life expectancy and intelligence. Patients who suffering from ectodermal dysplasia can be characterize variably. Multiple ectodermal derivatives can be affected, including teeth, hair, nails, sweat gland, etc [1]. The first case was described by Thurman in 1848 [2]. However, the medical term of ectodermal dysplasia was coined by Weech in 1929. Even though the birth prevalence of ectodermal dysplasia has been reported about 1 7 per 100,000, the exact number of ectodermal dysplasia is still unknown. The main reasons of ectodermal dysplasia are EDA genes mutations and X-linked recessive inheritance by parents, minor is deletion of certain genes. Ectodermal dysplasia has been classified more than 170 different subtypes [3]. Many cases of ectodermal dysplasia have been reported in US, but few cases have been reported among China population. Almost $91 \%$ of the ectodermal dysplasia patients accompanied with trichodysplasia, $80 \%$ with tooth agenesis, $75 \%$ with onychodysplasia, and $42 \%$ with dyshidrosis [4].
We hereby present a case report of a 5-year-old male child of China ethnicity with ectodermal dysplasia syndrome. Manifest the classical features and indentify the subgroup of the case presented.

\section{Case report}

A 5-year-old boy of China first reported to our hospital, with complaints of teeth missing and difficulty in chewing. No history of appearance of natal or neonatal teeth. The patient was the only child of his parents. Both his parents developed normally and had no history of ectodermal dysplasia syndrome in their families. However, his parents had consanguineous marriage. The medical history was unremarkable.

General examination found that the patient had broad depressed nasal bridge, short bulbous nose, sparse hair and eyebrows, which presented in (Figure 1-3). No deformities of the hands, fingers or ears were found.

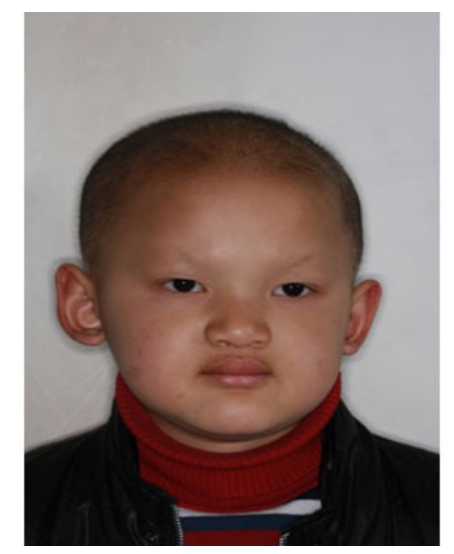

Figure 1: Anterior view. 


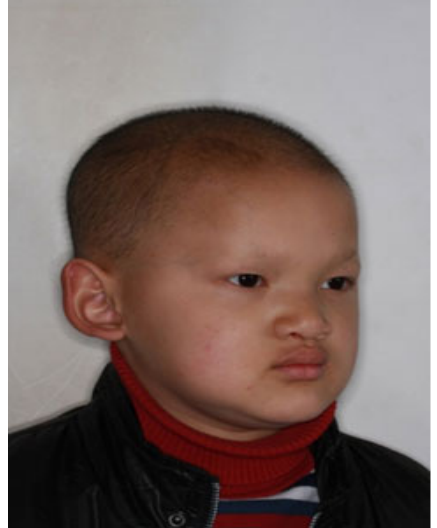

Figure 2: Profile view in 45 angle lateral phase.

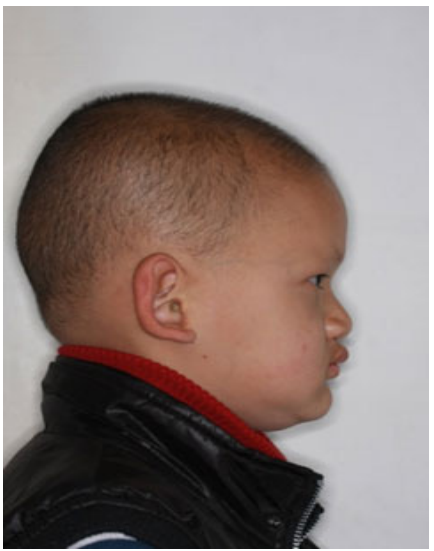

Figure 3: Profile view.

Intraoral examination revealed abnormal labial frenula. Wide space between 51 and 61 , which observed as small and conically crowned are presented in (Figure4-5). A majority of the deciduous teeth were shed, only three of which were retained in maxillary and mandibular anodontia that presented in (Figure 6-7). Most of the permanent tooth germ was missing. The alveolar process was found diminished. The lower arch showed considerably under developed in (Figure8).

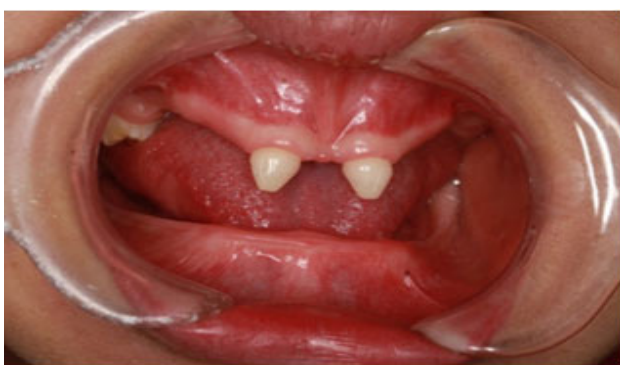

Figure 4: Wide space between 51 and 61.

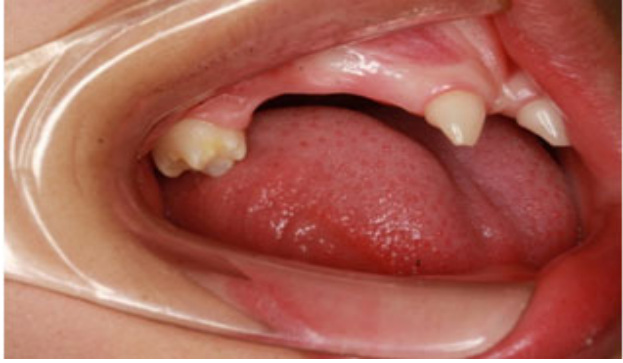

Figure 5: Abnormal labial frenum in maxillary anterior sulcus.

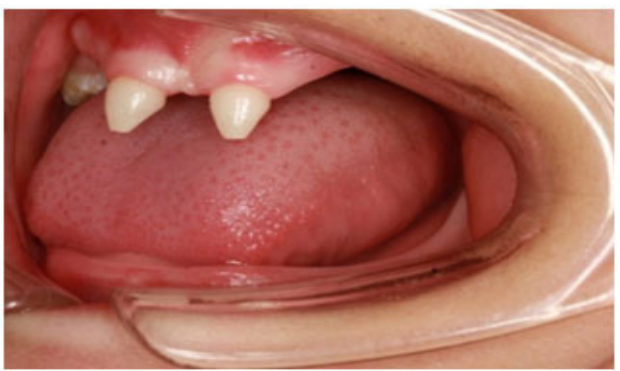

Figure 6: Small and conically crowned teeth irt 5A, 6A.

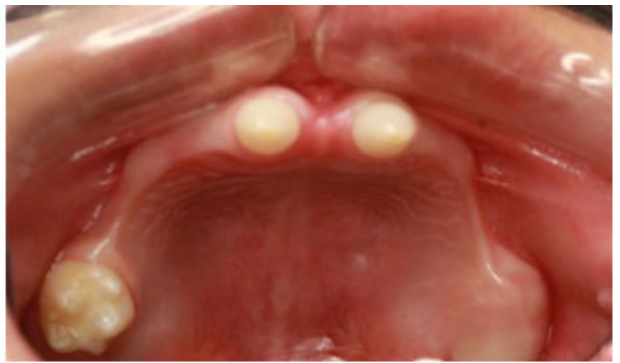

Figure 7: Clinically missing maxillary left primary molar teeth.

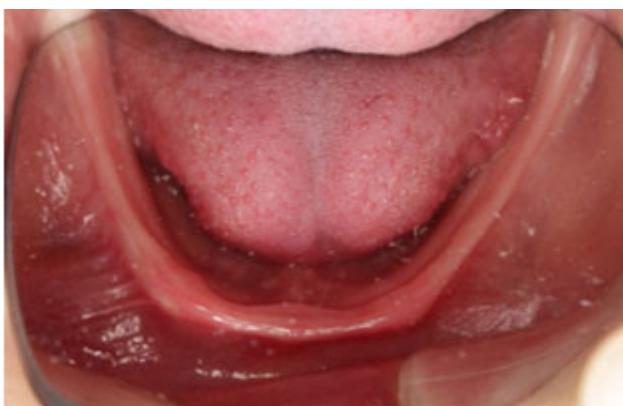

Figure 8: Under developed lower arch.

Based on the clinical and radiographic findings and subsequent medical examinations, the patient was diagnosed as ectodermal dysplasia. The patient and his parents singed the written informed consent and agreed to report the case. 
Due to personal reasons, occlusal reconstruction was the main purpose of the patient. A multidisciplinary treatment plan was designed by orthodonticsts, endodontists and prosthodontists $[5,6]$. First step, close the space between the maxillary primary central incisors by orthodontic therapy. Round Australian arch wires were used with mesialization mechanics to close the maxillary deciduous central incisor diastema and it was retained in position by giving a bonded retainer. Second step, fixed crowns repair short conical teeth. Third step, make a full mouth removable partial denture by prosthetics. Endosseous implants were an ideal option; however, the patient was too young and unable to afford the costs [7].

One year later, the space was closed by orthodontics treatment and clinically maxillary left and right first permanent molar erupted in (Figure 9-14). The results are presented in (Table 1).

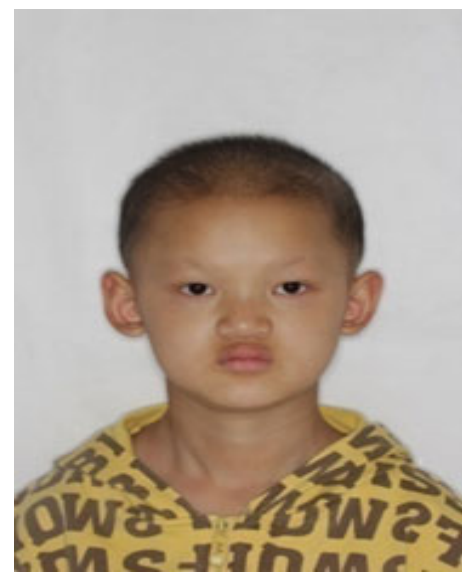

Figure 9: Anterior view (one year later).

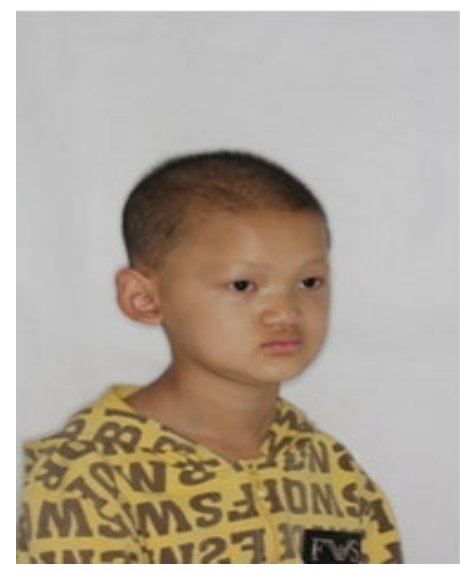

Figure 10: Profile view in 45 angle lateral phase (one year later)

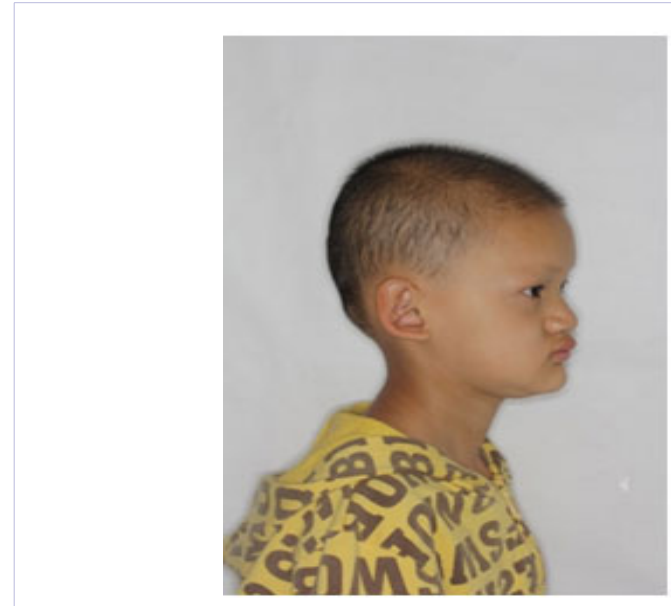

Figure 11: Profile view.

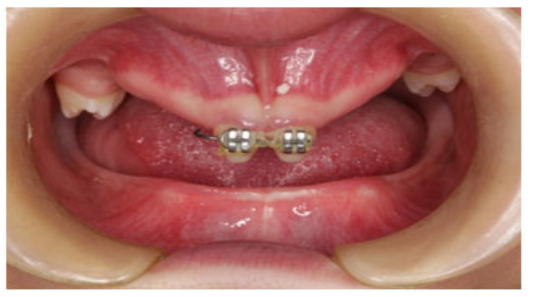

Figure 12: The space between 51 and 61 closed.

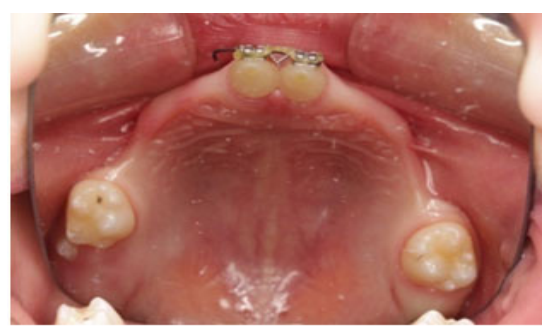

Figure 13: Clinically maxillary 16 and 26 erupted.

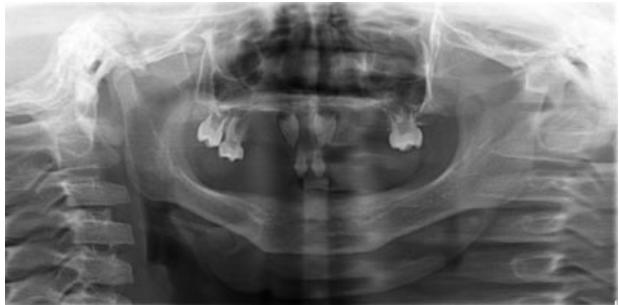

Figure 14: Panoramic view. 


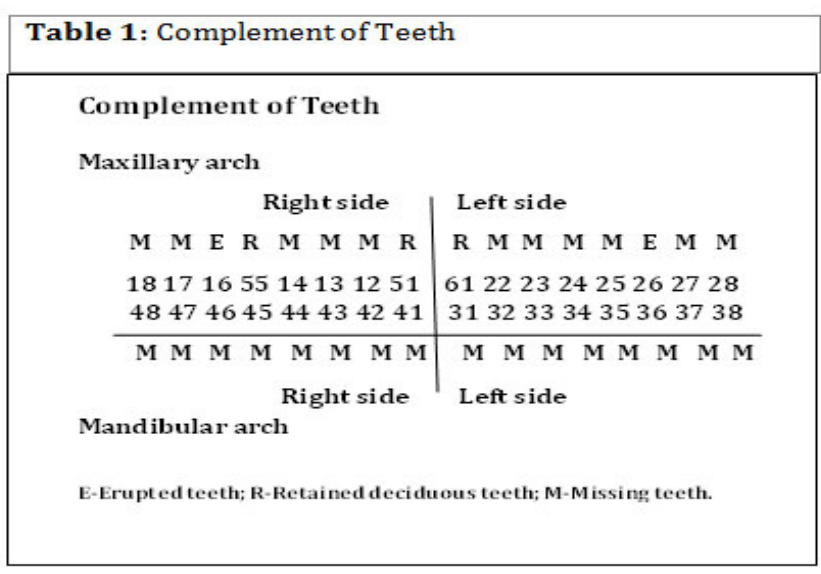

Based on the presence or absence types, ectodermal dysplasia syndrome can be classified into four primary presence subgroups [8]:

1: Trichodysplasia (Hair dysplasia),

2: Dental dysplasia

3: Onychodysplasia (Nail dysplasia)

4: Dyshidrosis (Sweat gland dysplasia)

The defects of the case presented can be categorized into Subgroup 1-2 [9].

\section{Discussion}

In present case report, most of the teeth were absent or abnormal morphogenesis. The patient presents with hypodontia, hypoplasia, retained deciduous teeth, and facial disfigurement, which highly suspected to be hypohidrotic ectodermal dysplasia [10]. Hypohidrotic ectodermal dysplasia, the most common type, seems to be X-linked inheritance pattern. The genes of hypohidrotic ectodermal dysplasia mapping to Xq12-q13, which makes males more susceptible than females, has identified to be a novel transmembrane protein(TNF family ligand and TNF receptor) [11]. TNF ligand and receptor, associated with epithelial mesenchymal interactions, play an important role in developmental regulate. During the initiation of development, these TNF ligands and receptors regulate ectodermal appendage formation and organogenesis [12].

Management of ectodermal dysplasia should consider both the demands of the patient and the treatment outcome, such as the costs of the treatment, the position of the remaining teeth in the arches, the volume of bone present, and age of the patient $[13,14]$. A multidisciplinary approach to ectodermal dysplasia involves many subjects, including oral physician, cardiologist, psychologist, geneticist, etc. Periodically dental counseling, strictly plaque control and oral hygiene maintenance can be helpful in prevention of dental decay. Fixed crowns can repair short conical teeth. Orthodontic intervention is necessary to correct the dental malocclusion. Space maintainers, for instance, can replace the congenitally missing teeth and improve aesthetics, speech and other physical functions of the patients. The final goal is to find a cost-effective treatment, minimize invasive risks and improve the life quality of the patients.

\section{Conclusion}

We hereby present an ectodermal dysplasia case report with usual oral findings such as fully edentulous in mandible and abnormal labial frenum in maxillary. A comprehensive treatment plan plays an important role in reconstruct occlusion and improves the life quality of the patients with ectodermal dysplasia syndrome.

\section{Acknowledgment}

This case report was surpported by the National Natural Science Foundation of China (81760193), Science and Technology Innovation Team Building Program of KMU(CXTD201607).

\section{References}

1. Jain D, Jain S, Kumar A and Rahangdale T. Hypohidrotic ectodermal dysplasia: prosthetic and endodontic management. Int J Clin Pediatr Dent. 2010;3(1):63-67. Doi:10.5005/jp-journals-10005-1056

2. Yenisey M, Guler A and Unal U. Orthodontic and prosthodontic treatment of ectodermal dysplasia: a case report. $\mathrm{Br}$ Dent J. 2004;196(11):677-679.

3. Hekmatfar S, Jafari K, Meshki R and Badakhsh S. Dental management of ectodermal dysplasia: two clinical case reports. J Dent Res Dent Clin Dent Prospect. 2012;6(3):108-112. Doi: 10.5681/joddd.2012.023

4. Holbrook KA. Structural abnormalities of the epidermally derived appendages in skin from patients with ectodermal dysplasia: insight into developmental errors. Birth Defects Orig Artic Ser. 1988;24(2):1544.

5. Badavannar AN, Manvi SF and Belludi A. Multidisplinary approach to ectodermal dysplasia: a case report. Int J Contemp Dent. 2011;2:7476.

6. Van Sickels JE, Raybould YP and Hicks P. Interdisciplinary management of patients with ectodermal dysplasia. J Oral Implantol. 2010;36(3):239-245

7. Guckes AD, Brahims JS, McCarthy GR, Rudy SF and Cooper LT. Using endosseous dental implants for patients with ectodermal dysplasia. J Am Dent Assoc 1991;122(10):59-62.

8. Ngan V and Writer S. Ectodermal dysplasia. Derm Net New Zealand. 2005

9. Suja J, George JC, Jose J and Alex KV. Multidisciplinary management of hypohydrotic ectodermal dysplasia - a case report. Clin Case Rep. 2015;3(5):280-286. doi: 10.1002/ccr3.209

10. Hekmatfar S, Jafari K, Meshki R and Badakhsh S. Dental management of ectodermal dysplasia: two clinical case reports. J Dent Res Dent Clin Dent Prospect. 2012;6(3):108-112. Doi: 10.5681/joddd.2012.023

11. Rajendran R and Sivapathasundaram B. Diseases of the skin. Shafer's text book of oral pathology. 2009;6:797-798.

12. Laurikkala J, Mikkola M, Mustonen T, Aberg T, Koppinen P, Pispa J, et al. TNF Signaling via the ligand-receptor pair ectodysplasin and edar controls the function of epithelial signaling centre and is regulated by Wnt and Activin during tooth organogenesis. Dev. Biol. 2001;229(2):443-455

13. Tarjan I, Gabris K and Rozsa N. Early prosthetic treatment of patients with ectodermal dysplasia: a clinical report. J Prosthet Dent. 2005;93(5):419-424.

14. Roshan Kumar P, Srivatsa G and Kashinath KR. Prosthodontic management of ectodermal dysplasia: a case report. Indian J Compr Dent Care. 2011;1:86-88. 